\title{
Nonclassical Cause of Obstructive Jaundice: A Case Report of Multiple Visceral Arterial Aneurysms in a Young Patient
}

\author{
Hassan Adnan Bukhari ${ }^{1,2}$, Noran Sultan², Waed Jameel ${ }^{2}$ \\ ${ }^{1}$ Department of Surgery, College of Medicine, Umm Al-Qura University, Makkah, Saudi Arabia \\ ${ }^{2}$ King Abdulaziz Hospital, Makkah, Saudi Arabia \\ Email: drhb2000@yahoo.com
}

How to cite this paper: Bukhari, H.A., Sultan, N. and Jameel, W. (2020) Nonclassical Cause of Obstructive Jaundice: A Case Report of Multiple Visceral Arterial Aneurysms in a Young Patient. Open Journal of Emergency Medicine, 8, 71-77. https://doi.org/10.4236/ojem.2020.83008

Received: July 2, 2020

Accepted: August 3, 2020

Published: August 6, 2020

Copyright $\odot 2020$ by author(s) and Scientific Research Publishing Inc. This work is licensed under the Creative Commons Attribution International License (CC BY 4.0).

http://creativecommons.org/licenses/by/4.0/

\begin{abstract}
Multiple visceral artery aneurysms are relatively rare; the rarest are superior mesenteric artery and gastroduodenal artery aneurysms. The clinical presentation depends on the size and site of the aneurysm. We report the case of a 33-year-old man with recurrent episodes of obstructive jaundice and weight loss. Computed tomography with contrast revealed scattered hepatic arterial aneurysms with two other aneurysms along the course of the left gastro-duodenal and superior mesenteric artery. Investigations to determine the etiology were negative, but the patient had a history of smoking and substance abuse. Angiography revealed an occluded feeding artery. The patient developed multiorgan failure, leading to cardiac arrest and died. Diagnosing multiple visceral artery aneurysms needs a high index of suspicion; early diagnosis enables early intervention, which can reduce the high mortality of this condition.
\end{abstract}

\section{Keywords}

Multiple Visceral Artery Aneurysms (MVAA), Gastroduodenal Artery Aneurysms (GDA), Hepatic Arterial Aneurysms (HAA)

\section{Introduction}

Multiple visceral artery aneurysms (MVAA) are relatively rare, ranging between $0.1 \%-0.2 \%$ of the total incidence of aneurysms. Among hepatic artery aneurysms, $60 \%$ are extra-hepatic, $30 \%$ occur in the right hepatic artery, and only $5 \%$ occur in the left hepatic artery. Superior mesenteric artery (SMA) and gastro- 
duodenal artery (GDA) aneurysms are considered rarest, comprising $1.5 \%$ of all VAAs [1] [2]. They can be defined as the presence of or more concomitant aneurysms in different arterial segments [3]. There are several risk factors leading to multiple aneurysms, with atherosclerosis being the main etiological factor [1] [3]. Other causes include polyarteritis nodosa, Behcet disease, neurofibromatosis, Marfan, Ehlers Danlos syndrome, and trauma [3] [4]. Idiopathic MVAA are restricted to the pediatric age group, but should not be overlooked as a possible diagnosis in adult patients [4]. Clinical presentations of aneurysms vary depending on their size and site; patients may also be asymptomatic. Significant risk factors are age above sixty years, male sex, a strong family history, hyperlipidemia, hypercholesterolemia, and cigarette smoking [1] [3]. Nonetheless, MVAAs can also occur in the absence of any risk factors [2]. It is essential to have a high index of suspicion when attempting to diagnose an arterial aneurysm [2]. Certain imaging modalities are crucial in the diagnosis of arterial aneurysms, such as contrast enhanced computed tomography (CT), which is considered the gold standard [3]. Treatment of MVAAs depends on the size, site, and extent of the disease [5]. Management options include percutaneous endovascular stenting and surgical repair [4].

We report the case of a young man presenting with jaundice due to MVAA.

\section{Case Presentation}

A 33-year-old male patient without a significant medical history presented to the emergency department complaining of sudden colicky epigastric pain radiating to the back that was relieved by leaning forward. The patient denied any history of nausea, vomiting, fever, bowel habit changes, or trauma, as well as significant weight loss and decreased oral intake over the last few months, A similar attack had occurred five months prior and had been relieved by painkillers, He had no previous surgical history; his family history was insignificant, including no history of hypertension or hyperlipidemia. He was a heavy smoker and abused several unknown substances.

On physical examination, the patient was hemodynamically stable but deeply jaundiced, the abdomen was soft, not distended, but there was tenderness in the epigastric area with no palpable mass felt. The rest of the examination was unremarkable.

Initial laboratory investigation revealed markedly elevated cholestatic enzymes alkaline phosphatase $1042 \mathrm{U} / \mathrm{L}$, AST $52 \mathrm{U} / \mathrm{L}$, ALT $63 \mathrm{U} / \mathrm{L}$, with normal amylase $55 \mathrm{U} / \mathrm{L}$, total bilirubin $11 \mathrm{mmol} / \mathrm{L}$. There was a mild elevation of white blood cell count and mild anemia. Differential diagnosis was obstructive jaundice secondary to common bile duct stone vs. periampullary tumor given the duration and the severity of jaundice clinically. The abdominal ultrasonography showed a distended gallbladder with a thick edematous wall of almost $6 \mathrm{~mm}$, filled with echogenic sludge and a single large calculus of $1.7 \mathrm{~cm}$ diameter with minimal pericholecystic free fluid. The common bile duct (CBD) was dilated with subtle intrahepatic dilatation. A large soft tissue structure $(5 \times 4.7 \mathrm{~cm})$ was 
noted in the pancreatic head. The CBD disappeared inside the head of the pancreas, most likely obstructed due to the intrapancreatic lesion.

The patient was admitted as a case of obstructive jaundice and underwent abdominal computed tomography angiogram (CTA). The CTA revealed multiple small-sized hepatic arterial aneurysms scattered along the right and left hepatic arteries, the largest one at the left hepatic lobe segment IV measuring $1.6 \mathrm{~cm}$. Two other aneurysms of $2 \mathrm{~cm}$ were seen along the course of the GDA/superior pancreaticoduodenal artery (PDA) surrounded by hypodense area (likely edema) inside the pancreatic head causing mass-effect on both pancreatic and biliary ducts (Figure 1).

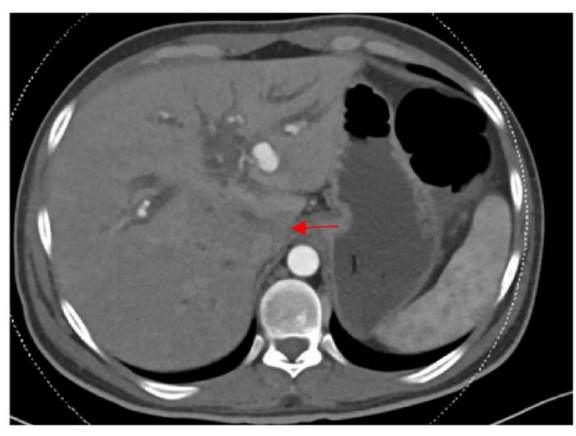

(a)

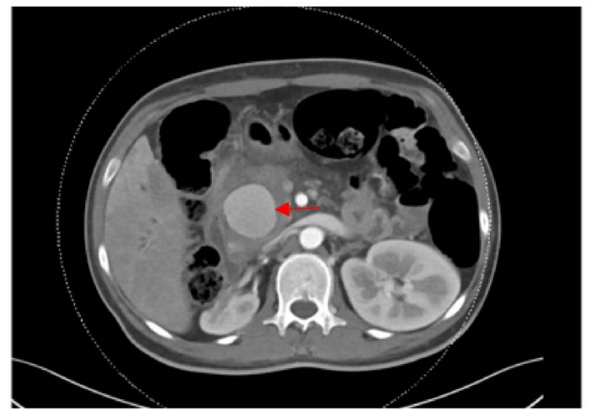

(b)

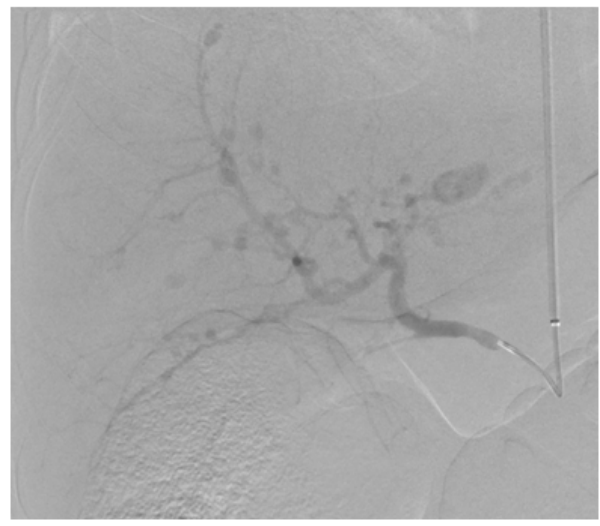

(c)

Figure 1. (a) Multiple intrahepatic aneurysms; (b) The CTA axial cut shows the largest aneurysm in the GDA/its branch inside pancreatic head with mass effect (red arrow); (c) 3DVR show multiple visceral aneurysms; the largest was related to the GDA. 
Small similar aneurysms of $3-4 \mathrm{~mm}$ seen along the SMA, inferior mesenteric artery, and splenic artery, along with few small aneurysms of the superficial femoral artery branches were noted. At the same time, the renal arteries on both sides, along with aortic and iliac artery were all spared. There was a small $1 \mathrm{~cm}$ non-enhancing cystic lesion at the tail of the pancreas and two soft tissue structures at the ilio-renal angle, the largest of which measured $4 \times 2.3 \mathrm{~cm}$, likely a splenule. Multiple intra-abdominal arterial aneurysm dilatations compatible with MVAA disease was the final diagnosis. The patient left the hospital against medical advice, but through our social works and the police service, we managed to reach him and convinced him to come back to the hospital after explaining how serious his condition is.

The patient was transferred and reevaluated by the vascular team at another tertiary hospital. The arrangement is made to undergo angiography for embolization. He started to deteriorate, so he was shifted urgently to the angiogram suite after intubation and sedation. Arterial access was established through the left radial artery. During the angiogram, the enormous aneurysm seen at the distal GDA did not opacify, most probably due to rupture that resulted in complete occlusion of the GDA/PDA by the surrounding hematoma (Figure 2) which explained why the patient deteriorated. The feeding artery (front door) was occluded using 2, 3-, and 4-mm coils with no immediate complications (Figure 3). Back door occlusion by obtaining SMA angiogram was not performed as the interventionist believed that occluding the feeding artery was enough at this stage. Post procedure, the patient was shifted to the intensive care unit and developed multiorgan failure, including severe pancreatitis confirmed by elevation of amylase post angioembolization. After 24 hours, he went into cardiac arrest for which cardiopulmonary resuscitation following the Advanced cardiac life support protocol was performed, but was not successful. He was pronounced dead after forty-five minutes.

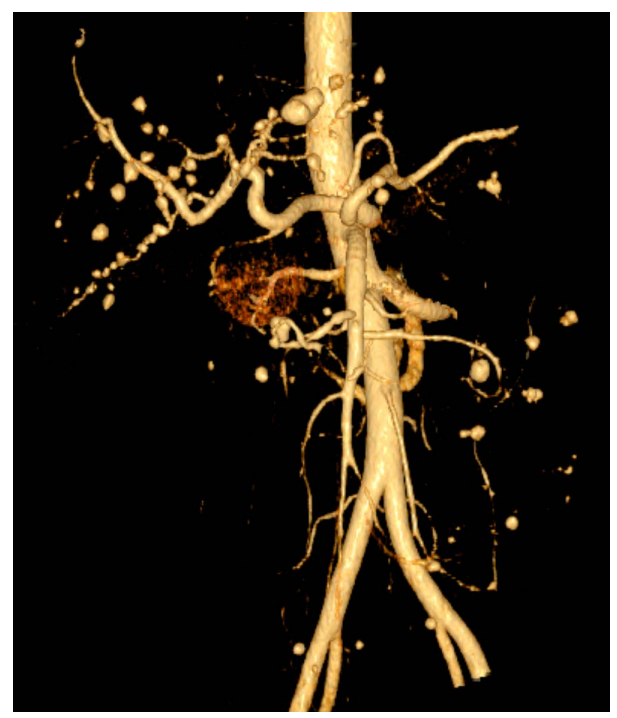

Figure 2. Angiogram showed multiple Intrahepatic aneurysms. 


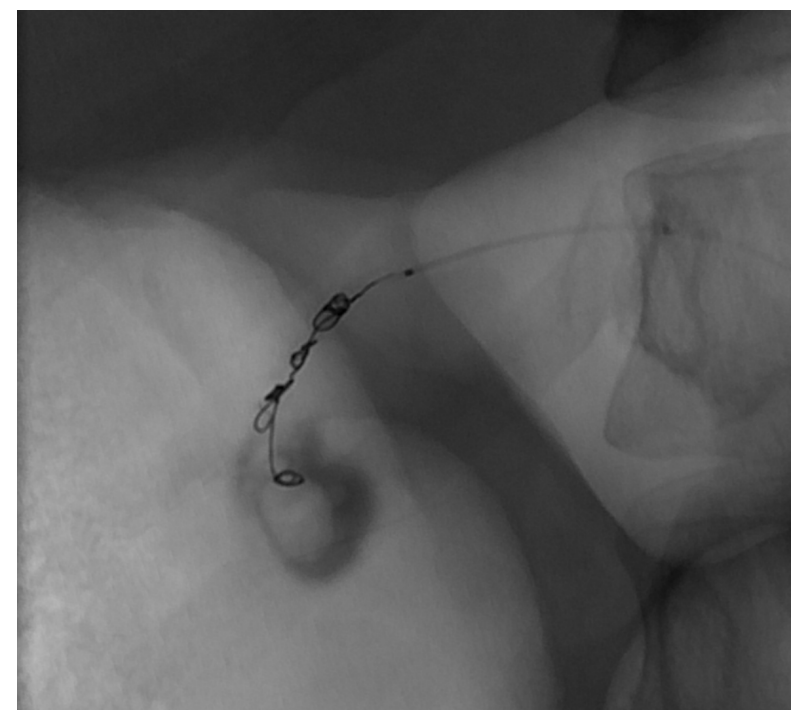

Figure 3. Angiogram with insertion of three coils, which are used to occlude the origin of GDA.

\section{Discussion}

Multiple visceral aneurysms are commonly seen in the elderly, which is mainly due to atherosclerosis. In our case, MVAA was diagnosed in a young healthy male. Studies have shown an increased prevalence among the male population [1] [4]. Furthermore, these aneurysms are usually associated with syndromes such as polyarteritis nodosa, Behcet disease, and Marfan syndrome [3] [4] [5]. Our patient tested negative for anti-nuclear and antineutrophil cytoplasmic antibodies and had no clinical features suggestive of Marfan syndrome or Behcet disease with no family history of genetic disorders. Blood cultures for fungal infection were negative, ruling out mycotic aneurysms. The lipid profile was normal, which rule out triglyceride-related pancreatitis. However, a strong history of smoking and drug abuse might explain the development of the disease in our patient.

The initial presentation of MVAA can be deceiving, such as in this case, who presented with symptoms suggestive of obstructive jaundice, which is due to GDA/superior PDA aneurysm [6]. This indicates how broad the manifestation of this disease could be. The rarity of the disease with a low index of suspicion will delay the diagnosis, which can lead to devastating outcomes like a rupture [1] [4]. Aneurysms have a high rate of complications such as spontaneous rupture and catastrophic bleeding especially if it was symptomatic or more then 2 $\mathrm{cm}$ [7]. In this case, there was a drop in the hemoglobin level, which was most likely due to retroperitoneal bleed, a bedside abdominal ultrasonography ruled out active intraabdominal bleeding [4] [5]. Rupture of GDA carry a high mortality rate that can reach $>70 \%$. Therefore, aneurysm of any size of this vessel or its branches like PDA should be managed once a diagnosis has been made to avoid devastating complications. During angioembolization, the interventionist should be aware of "front-door and back-door" approach. This means the embolization 
should be done at the origin of GDA (front-door) and angiogram of SMA \pm embolization of inferior pancreaticoduodenal artery (back-door) to prevent back flow bleeding from this artery. This technique ensures adequate control of both superior (from GDA) and inferior (from SMA) pancreaticoduodenal arteries. GDA embolization rarely cause major complication because the gastrointestinal tract has plentiful blood supply with extensive collaterals. In this case, could be one of the differential diagnosis for patient deterioration post-procedure is bleeding secondary to failure to control the back flow bleeding (back-door control). that ischemic complication can occur in $<15 \%$ [8]. Ischemic pancreatitis is hugely a rare complication post GDA embolization. There is one case reported post empirical GDA embolization for nonvariceal upper gastrointestinal bleed [9]. The patient went into profound shock with multi-organ failure due to severe pancreatitis following GDA embolization or from occlusion/thrombosis of the major pancreatic arterial supply. This could be the first case in the literature to discuss the rarity of MVAA with even more rare complications of GDA/PDA embolization.

\section{Conclusion}

We present this case to enrich the literature about this rare condition. MVAA is a challenging disease to diagnose initially due to its broad presentation, which depends on the site of the aneurysms. However, in young patients, it should be considered as a differential diagnosis as it could appear with no obvious risk factors. If an aneurysm found in one region, other arteries should be scanned. MVAA has a high rate of morbidity and mortality, moreover that necessity for proper and immediate management in cloud one of the option endovascular embolization [7], along with control of "front-door and back-door" feeding vessels will improve outcome. Failure to do that will result in poor bleeding control and increased risk of bleeding. Ischemic pancreatitis is a potential complication following GDA/PDA embolization and the treating physicians should be familiar with all complications related to this uncommon disease because it carries a very devastating poor outcome.

\section{Acknowledgements}

The authors would like to acknowledge the support provided by the staff of Radiology Department Al-Noor Specialist Hospital for providing the images used in this case, including our interventional radiologists Dr. Ahmed Haroun and Dr. Hossam Aljebali.

\section{Consent for Publication}

Consent was taken from the patient's next of kin.

\section{Conflicts of Interest}

The authors declared no conflicts of interest. 


\section{References}

[1] Sumpio, B., Eidt, J.F., Mills, S.R., et al. (2019) Overview of Visceral Artery Aneurysm and Pseudoaneurysm. Uptodate.

[2] Shawky, M.S., Tan, J. and French, R. (2015) Gastroduodenal Artery Aneurysm: A Case Report and Concise Review of Literature. Annals of Vascular Diseases, 8, 331-333. https://doi.org/10.3400/avd.cr.15-00086

[3] Trajbar, T., Pavic, P., Ivkosic, et al. (2006) A Rare Case of Multiple Aneurysms in a Young Patient. Collegium Antropologicum, 30, 235-238.

[4] Naha, K., Vivek, G., Shetty, R.K. and Dias, L.S. (2013) Multiple Idiopathic Arterial Aneurysms Masquerading as Aortic Dissection. BMJ Case Reports. https://doi.org/10.1136/bcr-2012-008030

[5] De Silva, W.S.L., Gamlaksha, D.S., Jayasekara, D.P. and Rajamanthri, S.D. (2017) A Splenic Artery Aneurysm Presenting with Multiple Episodes of Upper Gastrointestinal Bleeding: A Case Report. Journal of Medical Case Reports, 11, 123. https://doi.org/10.1186/s13256-017-1282-7

[6] Konstantakos, A.K., Coogan, S.M., Husni, E.A., et al. (2000) Aneurysm of the Gastroduodenal Artery: An Unusual Cause of Obstructive Jaundice. The American Surgeon, 66, 695-698.

[7] Ibrahim, F., Dunn, J., Rundback, J., Pellerito, J. and Galmer, A. (2018) Visceral Artery Aneurysms: Diagnosis, Surveillance, and Treatment. Current Treatment OPtions in Cardiovascular Medicine, 20, 97.

https://doi.org/10.1007/s11936-018-0696-x

[8] Barkun, A.N., et al. (2010) International Consensus Recommendations on the Management of Patients with Nonvariceal Upper Gastrointestinal Bleeding. Annals of Internal Medicine, 152, 101-113.

https://doi.org/10.7326/0003-4819-152-2-201001190-00009

[9] Chue, M.W., Venkatanarasimha, N. and Damodharan, K. (2017) Ischemic Pancreatitis: A Rare Complication of Empirical Gastroduodenal Artery Embolization. Indian Journal of Radiology and Imaging, 27, 338-341.

https://doi.org/10.4103/0971-3026.215571 